\title{
Expectativas de los alumnos de la UNMSM en relación al estudio del inglés como lengua extranjera
}

\author{
Minnie Lozada Trimbath y Alicia Alonzo Sutta \\ Departamento Académico de Lingüística
}

\section{Introducción}

IT 1 Perú es un país multilingüe donde se hablan el castellano, el quechua, 14 el aimara, y un alto número de lenguas amazónicas. Algunas de estas lenguas son adquiridas por determinados grupos como lengua materna o primera lengua y otras aprendidas en segundo término constituyen la segunda lengua de los hablantes. El número de hablantes de lenguas indígenas como primera lengua ha variado, presentando el castellano el mayor número de hablantes, debido a que éste es empleado como primera lengua y como segunda lengua en nuestro país. Dichas lenguas, como tales, cumplen una serie de funciones en la vida cotidiana de nuestros habitantess

Por otro lado, en el Perú se aprenden lenguas que llamamos extranjeras pues éstas no cumplen las funciones de comunicación en la población, ni tampoco son empleadas a nivel sociopolítico en la administración del país. Por ejemplo, el inglés y el francés son lenguas extranjeras en el Perú; sin embargo, remitiéndonos a otro contexto, en algunas ciudades del Canadá, el francés es lengua materna de gran parte de la población canadiense; pero para los residentes hablantes de inglés de esas mismas ciudades, el francés es su segunda lengua y no una lengua extranjera.

En el Perú hay distintos grados de presencia de lenguas extranjeras, por ejemplo, el inglés está bastante difundido. Si pensamos, por ejemplo, en el ambiente universitario vemos que en la Universidad Nacional Mayor de San Marcos los servicios para el aprendizaje de esta lengua presentan distintas modalidades. Una de ellas es la que se ofrece en algunas escuelas académicoprofesionales como un curso que forma parte del currículo, teniendo éstos dis- 
tinto carácter, en algunos casos, como curso electivo, y en otros, como curso obligatorio. Independientemente del currículo, también, se ofrecen cursos cortos de idiomas a los alumnos interesados en seguirlos. Otra modalidad es la que ofrece el Centro de Idiomas de la Facultad de Letras y Ciencias Humanas, el cual se desarrolla independientemente del plan de estudios de las Escuelas. Aquí el estudiante, alumno de la universidad o de fuera de ella, necesita desplegar un esfuerzo continuado para ir avanzando en el control y dominio de la lengua desde un nivel básico hasta uno avanzado.

El presente estudio tiene como propósito explorar y describir las expectativas del alumno universitario de San Marcos respecto a los cursos que le ofrecen en un Centro de Idiomas para aprender una lengua extranjera, el inglés.

¿Qué anima a los alumnos a realizar el esfuerzo de estudiar inglés por su cuenta? La respuesta, nos dirán algunos, es que necesitan aprender la nueva lengua para sus estudios y esperan que el centro los ayude a hacerlo. La necesidad existe, pero qué sabemos sobre la orientación de la energía y disposición con que se acercan los alumnos a este aprendizaje. Por otra parte, las razones o motivos que mueven a los alumnos a realizar el esfuerzo de matricularse en un centro de idiomas pueden ser varias. ¿Son éstas siempre de carácter práctico? ¿Es la necesidad de consultar bibliografía durante sus estudios universitarios la que los mueve? ¿Hay exigencia en este sentido en nuestras Facultades? Pero, podría haber otras respueștas, iquizá haya ahumnos a guienes, por ejemplo, les interesa conocer y descubrir la estructura de la lengua a esto es, el estudio de la lengua por la lengua en sí y no para un fin utilitario.

¿Qué esperan que les ofrezca el centro? Lo que un alumno espera de un Centro de Idiomas dependerá de los fines que él tenga y de su concepción del aprendizaje. Hay en este momento planteamientos nuevos respecto de la manera de orientar a los alumnos para que aprendan. ¿Hasta qué punto los estudiantes que se matriculan en un centro para aprender otra lengua esperan cambios con respecto a lo que tradicionalmente se ha venido haciendo en otras instituciones? ¿Querrán, por ejemplo, seguir cursos en los que el profesor cumpla con los libros correspondientes a una serie determinada al pie de la letra, o en donde se enfatice la gramática? Este estudio nos permitirá conocer, entonces, algo más sobre la manera de pensar y sentir de los estudiantes de una lengua extranjera y así entender mejor el tipo de servicio que el usuario del Centro de Idiomas de la Facultad de Letras espera recibir. 


\subsection{Planteamiento del problema}

Hay poco material bibliográfico sobre motivación y expectativas en la enseñanza de segunda lengua en el Perú. Existe un estudio sobre motivos o razones por las que se estudia inglés en la secundaria estatal de Lima realizado por PozziEscot y Lozada (1989). Los resultados mostraron que los alumnos, en lo referente a orientación instrumental o de carácter utilitario, elegían aprender lengua con el fin de viajar, obtener mejor información, conseguir un buen empleo y finalmente lograr mayor aceptación social. En lo que se refiere a orientación integracionista, las razones por las que los alumnos manifestaban aprender inglés eran las siguientes, de mayor a menor importancia: comprender a la gente de habla inglesa, tener amigos entre la gente de habla inglesa, ir a vivir a un país de habla inglesa y en último lugar pensar y comportarse como gente de habla inglesa.

Otro trabajo es el realizado por Lozada, Alonzo y Reyes (1997), en tres Escuelas Académico-Profesionales de San Marcos, sobre la percepción del estudiante universitario en relación a la utilidad de conocer idiomas. Uno de los aspectos estudiados en dicha investigación, el relativo a las habilidades lingüísticas preferidas por los alumnos, se puede relacionar con las expectativas que ellos pueden tener respecto del aprendizaje de idiomas. Los resultados, viéndolo desde el punto de vista de lo que es útil para la época de estudios, muestran un interés de los alumnos de tercer año (sexto ciclo) no sólo por la comprensión de lectựa sinōtámbién poela comprensión oral. Entre los alumnos de quinto año (décimo ciclo) se mantieneel interés antes mencionado aunque en un porcentaje significativo se añade el de conversar en la lengua extranjera. En otras palabras, la lengua oral está presente entre las preferencias de los alumnos. Cuando se pide a los estudiantes que opinen pensando en su vida profesional futura, destaca otro aspecto: se considera necesario manejar a la vez no una, sino varias habilidades lingüísticas.

\subsection{El inglés y el contexto sociolingüístico}

Como ya hemos señalado, en el Perú hay distintos grados de presencia de lenguas extranjeras, por ejemplo, el inglés está bastante difundido. Si pensamos, por ejemplo, en el ambiente universitario vemos que éste es muy exigente para quien quiere permanecer actualizado. Los estudiantes son personas muy hábiles y en su mayoría están acostumbrados al estudio y al esfuerzo de todo tipo y muchos reconocen la necesidad de aprender lenguas extranjeras, de prefe- 
rencia el inglés, aunque hay muchos también interesados en el francés De las lenguas nativas la que atrae más el interés de los estudiantes es el quechua. (Lozada, Alonzo y Reyes, 1997)

¿Cuál es el papel que cumple el inglés en el medio universitario? Dentro del país, permite el acceso a revistas y libros científicos recientes, a documentos variados y a la posibilidad de conversar a través de Internet; así mismo la posibilidad de escuchar ponencias de visitantes extranjeros y videoconferencias en inglés, entre otros. Además, para el estudiante que desea realizar estudios en países de habla inglesa, es necesario poseer un nivel de dominio avanzado de la lengua.

Fuera de la universidad, uno de los medios para conseguir información y estar actualizado es la televisión por cable. Algunos de los canales que se ven en nuestro país tienen toda su programación en inglés.

En el campo del trabajo, el saber inglés constituye un requisito más para alcanzar una colocación en una serie de empleos. Además es una lengua indispensable en algunas especialidades, por ejemplo, en distintas áreas del turismo o en la traducción o interpretación.

Los sujetos de esta investigación son personas que inician sus estudios de inglés en un centro de idioninas: Alrespectō hay un punto que se debe tener en cuenta: los estudiantes que llegan al centro después de cinco años de estudios de secundaria deberían traer una base de inglés, pero sabemos que la escuela secundaria estatal no les ha ofrecido a la mayor parte de ellos la posibilidad de un aprendizaje adecuado de dicha lengua. Según Williams y Burden (1997: 129) las experiencias previas influyen en las creencias iniciales que los alumnos tienen sobre su capacidad para aprender. Podría suceder entonces que una institución que se dedica a la enseñanza de esta lengua no sólo tenga que ayudar al alumno en su aprendizaje del inglés, sino que también tenga que ayudarlo a confiar en su capacidad para aprender dicha lengua.

\subsection{Marco teórico}

En esta sección vamos a hacer la distinción entre algunos términos. En primer lugar, no entendemos como sinónimos a términos frecuentemente usados como tales, como son los de motivación y orientación. La orientación es un término empleado por Gardner y Lambert en sus estudios de motivación en el 
Canadá. Cuando hablamos de orientación nos referimos a las razones o motivos que tiene un estudiante para aprender una segunda lengua.

Gardner y Lambert distinguen entre una orientación integracionista y una orientación instrumental. La orientación integracionista considera el acercamiento a la otra cultura, hasta la integración total en ella. La instrumental, en cambio, se refiere a las razones de carácter práctico para aprender lengua

Orientación no es igual a motivación sino que representa razones para estudiar la lengua. Una orientación integracionista ocurre cuando el que aprende está estudiando una lengua por un deseo de identificación con la cultura de los hablantes de dicha lengua. Una orientación instrumental describe un grupo de factores relacionados con la motivación que surge de objetivos externos tales como pasar exámenes, recompensas financieras, perfeccionarse en una carrera o conseguir una promoción (Williams y Burden,1997: 115)

La información de los primeros resultados de investigación sobre este tema, en el Canadá, indicaba que los alumnos que tenían orientación integracionista mostraban un rendimiento mayor en el aprendizaje de la segunda lengua, en ese caso, del francés. Se podría pensar que la orientación en el caso de una lengua extranjera sería más de tipo instrumental o utilitario. Esto es lo que ha sucedido en algunas investigaciones realizadas en otros contextos. Por ello, Gardner (Williams, 1997: 117) aclara quela importancia de una u otra brientación parece depender de los diferentes contextosengque secapiende la nuevalengua. En el presente estudio, vamos a usar indistintamente los términos lengua extranjera y segunda lengua para el inglés en el Perú, recordando que éste no es usado en la vida cotidiana e institucional de la población en general.

Los alumnos que se matriculan en centros de idiomas, generalmente pertenecen a distintas especialidades. Algunos de ellos se preparan para especialidades en donde el estudio sistemático de la lengua y el trabajo con ella es lo primordial. Estos especialistas serían los siguientes: Lingüistas, profesores de idiomas formados en centros de educación superior, traductores e interpretes y profesores de lengua materna, castellana, entre otros.

Por lo antes mencionado podemos decir que la mayoría de los alumnos de un centro de idiomas, no son especialistas en lengua, y, según el contexto socio-cultural en que se hayan formado y su experiencia previa con el inglés en el colegio, traen algunas ideas sin base científica sobre la lengua y sobre su 
aprendizaje. Es a este punto al que se refieren Richards y Lockhart cuando nos hablan de los sistemas de creencias de los estudiantes y su importancia.

"Los sistemas de creencias de los alumnos abarcan una amplia gama de puntos y pueden influir en la motivación de los alumnos para aprender, en sus expectativas acerca del aprendizaje de lengua, sus percepciones sobre lo que es fácil o difícil en una lengua, tanto como el tipo de estrategias de aprendizaje a favor de las cuales están". (Richards y Lockhart, 1994: 52)

Pensamos que el sistema de creencias respecto del aprendizaje de una segunda lengua es diferente entre un escolar de secundaria y un universitario o profesional. Es posible que los fines para los cuales se necesita la lengua influyan en una revisión de las creencias. Dichos fines pueden ser de carácter académico, laboral o de comunicación general. Según sea el caso, se considerará, por ejemplo, que es necesario prepararse para desarrollar ciertas habilidades lingüísticas orales y/o escritas, que dichas habilidades conviene practicarlas en relación a ciertos temas que son más prioritarios que otros, que los profesores deben emplear determinada metodología, ete.

\section{Metodología}

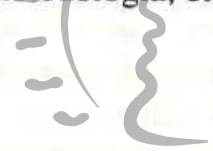

El diseño de la investigación realizadacorresponde a un estudio de casos con fines, principalmente de exploración y despripción . El caso seleccionado es el del alumnado del Primer Ciclo del Nivel Básico del Centro de Idiomas de la Facultad de Letras de la Universidad Nacional Mayor de San Marcos. Se ha estudiado a la población que cursaba el primer ciclo en el mes de septiembre del año 1998, mes que corresponde al segundo semestre de estudios en la universidad. Esto nos permitió obtener información de un grupo que estudia inglés paralelamente a sus estudios universitarios de especialidad. Es necesario destacar el esfuerzo que realizan los alumnos que estudian idiomas paralelamente a sus estudios de especialidad. Cabe señalar que los estudiantes encuestados tenían pocas semanas de asistencia a la institución y por ello no estaban tan influidos por ella.

Para realizar nuestro estudio, consideramos, como variable independiente, el área de actividad: estudios o trabajo, a la que se dedica la población total. La variable dependiente será la expectativa que el alumno tenía para aprender idiomas en el Centro de Idiomas de la Facultad de Letras.. 
Población. Los sujetos del estudio, como ya se ha indicado, pertenecen al Centro de Idiomas de la Facultad de Letras y Ciencias Humanas de la UNMSM. Dicho centro ofrece programas de aprendizaje de lenguas para los estudiantes de las diversas especialidades de la universidad así como para el público interesado en general.

Entre las lenguas que el Centro ofreció el año de la investigación (1998) se cuentan, entre otras, el inglés, el francés y el quechua, lenguas que al inicio planificamos en nuestra estudio para conocer la motivación de los alumnos que eligieron dichas lenguas; así como las expectativas que tenían de las mismas. A lo largo del año, el curso de la lengua quechua no se realizó. Independientemente de esta situación, al comenzar las primeras acciones programadas llegamos a la conclusión de que, siendo diferente el contexto y los papeles que cumplen las lenguas mencionadas en nuestra sociedad, se necesitaría un mayor tiempo de aquél con que contábamos para realizar un estudio adecuado. Por ello, nos vimos en la necesidad de orientar nuestra investigación al conjunto de mayor población, el de los alumnos que estudiaban el idioma inglés.

El curso de inglés se ofreció en el centro en dos modalidades, intensiva y regular, las cuales se diferencian por el número de horas del dictado del curso. La modalidad regular, en la época en que se aplicó la encuesta, tenía alumnos matriculados solamente en el horario del día sábado. Debemos señalar que en el planteamiento de nuểstra investigación no se hątomado en cuenta las diferencias que pudieran, existir entre los grupos de alumnos del ciclo regular e intensivo, por lo que no se ha realizado clasificaciones bajo esta variable, sin embargo pensamos que una futura investigación debería tener en cuenta este criterio.

La comunidad lingüística comprendida en el tema de nuestra investigación está constituida por aquellos alumnos que inician sus estudios de una lengua extranjera -inglés- en el Centro de Idiomas. Los alumnos encuestados estuvieron distribuidos en seis secciones correspondientes al primer ciclo del nivel básico, los que hicieron un total de 77 . Además de éstos, se cuenta un número de 23 alumnos que habiendo asistido al curso no se encontraron presentes en el momento de la aplicación de la encuesta.

Creemos importante destacar algunas características de los alumnos estudiados, puesto que éstas nos permitirán conocer de una manera aproximada el tipo de alumno que estudia en el Centro de Idiomas. La información obtenida 
se refiere a la edad, la especialidad que estudia el alumno, y al trabajo que realiza simultáneamente a los cursos de idiomas, cuando así ocurre.

Entre los alumnos encuestados del centro encontramos que 44 de ellos son estudiantes de pregrado de distintas especialidades de nuestra universidad y complementariamente 33 se dedican a otras actividades como estudios en otras universidades, trabajadores, profesionales que trabajan en distintas instituciones y jóvenes egresados de secundaria que aún no estudian ni trabajan (sólo uno se estaba preparando para ingresar a la universidad). Los sujetos mencionados constituyen la comunidad linguística que estaba cursando estudios de primer ciclo del nivel básico en el mes de septiembre del año antes mencionado. Ver Gráfico $\mathrm{N}^{\circ} 1$.

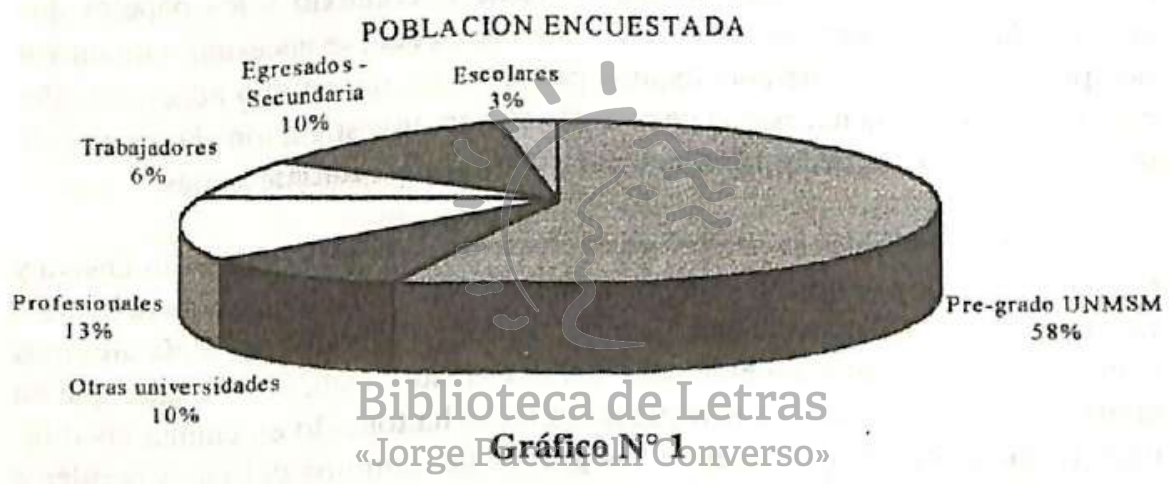

- ESTUDIANTES DE PREGRADO DE LA UNMSM. El mayor número de alumnos es el que proviene de las distintas especialidades de la UNMSM. Este asciende a 44 y sus edades oscilan entre 17 y 35 años, concentrándose mayormente entre 21 y 24 años.

- ESTUDIANTES DE OTRAS UNIVERSIDADES. Los alumnos provenían de las universidades Femenina, San Martín de Porres, Agraria, Federico Villarreal y la del Callao. El número de alumnos asciende a 8 y sus edades estaban comprendidas entre 18 y 28 años.

- PROFESIONALES. El número de profesionales que iniciaba sus estudios de inglés ascendía a 10. Ellos pertenecían a las siguientes especialidades: profesor (uno de los cuales es cesante), abogado, periodista, odontólogo, contador, trabajador social. Sus edades estaban comprendidas entre 26 y 35 años. 
- TRABAJADORES. Habían cinco trabajadores entre los cuales contamos a vendedores, asistentes de oficina y de tienda, y enfermeras, cuyas edades fluctuaban entre 17 y 38 años.

- EGRESADOS DE SECUNDARIA. EI Centro contaba con 8 estudiantes egresados de la secundaria, uno de los cuales asistía al Centro Preuniversitario de San Marcos. Los otros no estudiaban ni trabajaban aún. Sus edades oscilan entre 17 a 24 años.

- ESCOLARES. También encontramos a dos estudiantes escolares. Nuestro estudio está centrado principalmente en los 44 alumnos del Primer Ciclo del Nivel Básico del Centro de Idiomas de la Facultad de Letras y Ciencias Humanas. Lo que se presenta, a continuación, se basará, principalmente, en datos de este grupo. Consideramos de interés tener en cuenta, también, los datos de 31 alumnos de los otros grupos que asisten al centro de idiomas: 8 estudiantes de otras universidades, 10 profesionales, 5 trabajadores, y 8 egresados de secundaria que aún no trabajan ni estudian una carrera. No incluimos a 2 escolares por ser muy bajo su número. Debido a que el número de cada uno de los grupos es bastante reducido, lo cual no permite una comparación adecuada, cuando se haga referencia a estos 31 alumnos (menos los dos escolares omitidos) sólo se mencionarán algunas características. En adelante, nos referiremos a éstos como "los otros alumnos del centro de idiomas"

Los alumnos de p̄regradoude ŚanlMiaroos/que cursan el primer ciclo del nivel básico provienen de distintas especialidades. Ver Gráfico $\mathrm{N}^{\circ} 2$.

ESPECIALIdAd dE LOS ALUMNOS DE PREgRAdO - CICLO I - CENTRO dE IDIOMAS

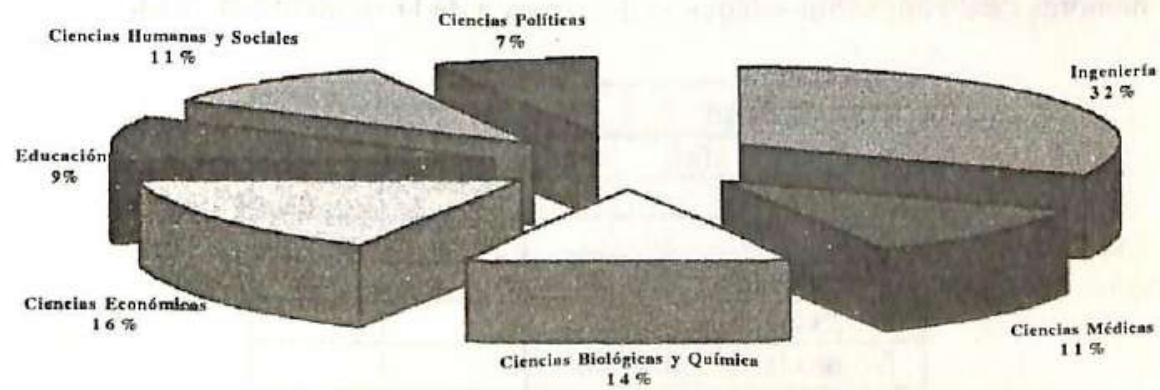

Gráfico $\mathrm{N}^{\circ} 2$ 
- Ingeniería y Matemática. Es el área que presenta un mayor número de estudiantes universitarios de pregrado. En ella están comprendidas las especialidades de Estadística, Física, y las de Ingeniería Electrónica, Mecánica, Industrial, Química, Geográfica, entre otras. En términos porcentuales, este grupo alcanza un 32\% (14 sujetos) del primer ciclo básico.

- Ciencias Económicas. Este grupo está constituido por los universitarios de Administración de Empresas, Economía y Contabilidad, alcanzando un porcentaje de $16 \%$ ( 7 sujetos) de la población estudiantil de idiomas en el centro.

- Ciencias Biológicas. Se cuentan las especialidades de Química, Farmacia y Bioquímica, así como las de Ciencias Biológicas. El conjunto está formado por 6 sujetos.

- Ciencias Médicas. Hemos clasificadoa las especialidades de la salud dentro de esta área, dentro de la que se encuentran Odontología, Nutrición y Medicina Humana. El conjunto es de 5 sujetos.

- Ciencias Humanas y Sociales. El conjunto está formado por 5 sujetos.

- Ciencias de la Educación. Son 4 los sujetos, dos de las especialidades de Biología y Químiea, y dos de laespecialidad de Ciencias Sociales.

\section{"Jorge Puccinelli Converso"}

- Ciencias Políticas. El conjunto está formado por 3 alumnos.

Edad. En lo relativo a la edad, la mayoría de los alumnos de pregrado de San Marcos que iniciaban sus estudios de inglés en el Centro de Idiomas eran menores de 29 años. Sus edades se distribuyen de la siguiente manera:

\begin{tabular}{|l|c|}
\hline \multicolumn{1}{|c|}{ Edad } & $N^{\circ}$ de Sujetos \\
\hline De 17 a 20 años & 6 \\
\hline De 21 a 24 & 27 \\
\hline De 25 a 28 & 8 \\
\hline De 29 a 32 & 1 \\
\hline De 33 a 35 & 1 \\
\hline No dio la información & 1 \\
\hline
\end{tabular}




\subsection{Instrumentos}

El instrumento empleado fue un cuestionario compuesto de una primera parte, en la que se consideran preguntas abiertas sobre datos personales del alumno, y de una segunda parte, con preguntas estructuradas siguiendo el método de Likert. Éste propone que se presente un enunciado al alumno entrevistado, en el cual deberá manifestar su posición al respecto, procediendo a elegir una de cinco categorías de respuesta: Muy de acuerdo, de acuerdo, indeciso, en desacuerdo, muy en desacuerdo. (Babbie, 1988: 320)

Las preguntas del cuestionario se elaboraron tomando en cuenta la situación particular que se investigaba: alumnos que inician sus estudios de inglés en un centro de idiomas dentro de una universidad estatal. En este punto estamos de acuerdo con Gardner en que "no hay una Batería de Exámenes de Actitud/Motivación, sino que los ítemes deberían desarrollarse de modo que sean apropiados para la situación particular investigada". (Gardner, citado por William y Burden, 1997: 116)

Como paso previo a la elaboración del cuestionario que se iba a aplicar a los alumnos, además de consultar bibliografía sobre el tema, en el mes de mayo se realizó un sondeo escrito mediante un cuestionario, entre 39 alumnos de tres secciones (grupos de 15, 9 y 15 estudiantes) del Centro de Idiomas de la Facultad de Letras y Ciencias Humanas. Dicho sondè tenía por finalidad recoger mediante preguntas abiertas, resueltas por-escrito, la opinión de los alumnos sobre su motivación, orientación y expectativas con relación al aprendizaje del inglés. Luego del análisis y categorización de las respuestas de los estudiantes, se seleccionaron varias de ellas para incluirlas como preguntas del cuestionario. Por otra parte, la consulta bibliográfica de Williams y Burden (1997), Richards y Lockhart (1994), y Pozzi-Escot y Lozada (1989) sirvieron de fuente para incluir varias preguntas en el cuestionario.

Luego de elaborar el cuestionario se hizo una aplicación piloto a 42 alumnos de tres secciones ( 16,13 y 13 estudiantes) del Centro de Idiomas, todos del segundo ciclo. Basándonos en las dudas de los estudiantes, para el cuestionario definitivo se cambiaron algunos preguntas (ítemes), en especial en la sección Datos Personales. Además se modificaron los ejemplos de las instrucciones generales.

El cuestionario definitivo quedó estructurado de la siguiente manera: 


\begin{tabular}{|c|c|}
\hline I. DATOS PERSONALES & PREGUNTAS \\
\hline Items & 01 a 07 \\
\hline $\begin{array}{ll}\text { Total de preguntas } \\
\end{array}$ & 07 preguntas \\
\hline \multicolumn{2}{|c|}{$\begin{array}{l}\text { II. VARIABLE: ORIENTACIÓN (razones por las que el inglés es necesario) } \\
\text { Factores: }\end{array}$} \\
\hline $\begin{array}{l}\text { - Desempeño como estudiante } \\
\text { - Desempeño laboral/profesional } \\
\text { - Actualización } \\
\text { - Viaje } \\
\text { - Integración y cultura } \\
\text { - Influencia significativa }\end{array}$ & $\begin{array}{l}18,19 \\
20,25 \\
23 \\
21 \\
22,24,26 \\
27 \\
\end{array}$ \\
\hline \begin{tabular}{|c|} 
Total de preguntas \\
\end{tabular} & 10 preguntas \\
\hline \multicolumn{2}{|c|}{$\begin{array}{l}\text { III. VARIABLE: EXPECTATIVAS EN RELACIÓN AL APRENDIZAJE } \\
\text { DE LA LENGUA }\end{array}$} \\
\hline $\begin{array}{l}\text { - Nivel de dominio del aprendizaje } \\
\text { - Interés sobre determinados temas y } \\
\text { habilidades como contenidos de los cursos } \\
\text { - Interés por técnicas metodológicas }\end{array}$ & $\begin{array}{l}28,31 \\
29,30,32,33,34,38 \\
35,36,39,40,41,42,43 \\
44,4537\end{array}$ \\
\hline Total de preguntas & 18 preguntas \\
\hline
\end{tabular}

\section{Resultados}

Las respuestas dejôs sujetos estudiảdos que se presentan a continuación están organizadas de acuerdo a las variables consideradas en la investigación, de ahí que esta sección comprende dos partes: orientación y expectativas.

\subsection{Orientación}

En esta sección se revisan las razones que mueven a los alumnos a realizar estudios de inglés considerándolas desde dos perspectivas, una de carácter utilitario llamada instrumental, y otra de acercamiento e identificación con la cultura llamada integracionista.

\subsubsection{Orientación instrumental}

\subsubsection{El inglés para el estudio}

Esta sección tiene en cuenta lo útil de saber inglés para incrementar, complementar o consolidar la formación académica del alumno; así mismo, para 
contar con información de diversa índole que considere importante, aún cuando ésta no sea actualizada

Item 18: El saber inglés me dará la posibilidad de acceder a mayor información bibliográfica.

Cuando nos referimos a información bibliográfica debemos tener en cuenta que la lectura, ahora, se realiza no sólo en libros, revistas y otros documentos, sino también en Internet.

Item 19: Saber inglés es útil porque algunos eventos académicos se realizan en idioma extranjero.

Nos parece conveniente señalar que los mismos alumnos plantearon el tema del ítem 19, pues normalmente los que trabajamos en la enseñanza de lenguas extranjeras consideramos que es el material impreso el que necesita poder manejar el alumno universitario. Pero, también es cierto que hay algunos eventos académicos en los que el ponente extranjero emplea el inglés, y si no se conoce dicha lengua depende uno del intérprete.

Respecto a la utilidad del inglés para acceder a la bibliografía (ítem18), el acuerdo es total. por parte de los estudiantes de pregrado; obsérvese que un $79.5 \%$ de ellos está "muy dejacuerdo" con el enuneiado y un $20.5 \%$ manifiesta estar "de acuerdo", lo cual arroja un resultado de aceptación general que asciende al $100 \%$ de la población entrevistada (Ver Gráfico 3).

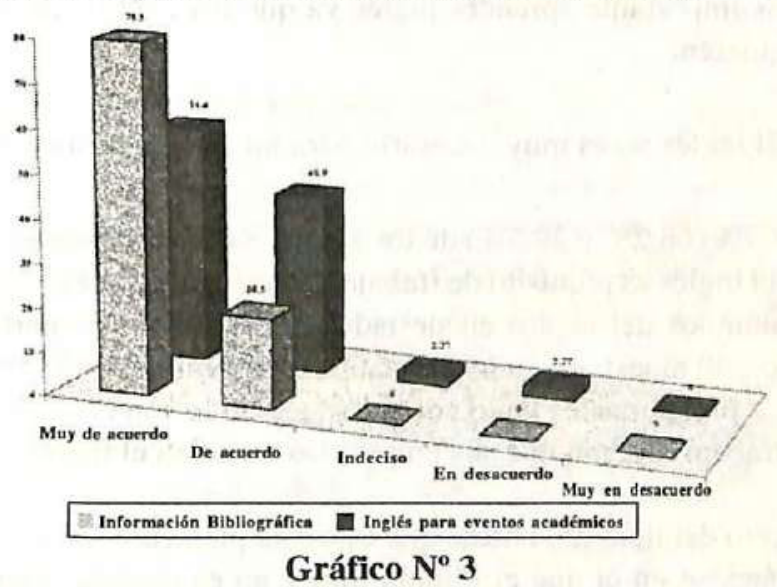


En cuanto al inglés y los eventos académicos (ítem 19 ), un $95.5 \%$ expresa acuerdo (54.6\% "muy de acuerdo" y $40.9 \%$ "de acuerdo"). Sólo dos alumnos piensan de manera diferente.

Los otros alumnos del centro encuestados que no son estudiantes del pregrado se pronuncian respecto de las preguntas de manera semejante a los alumnos de San Marcos. De los 31 sujetos, sólo 2 no manifiestan acuerdo (un trabajador y 1 egresado de secundaria).

Por lo tanto podemos inferir que el inglés, para el desempeño estudiantil y para los eventos académicos, es considerado por todos como útil. Lo que faltaría profundizar es cuáles son las actividades lingüísticas de los eventos académicos. Estos podrían incluir la lectura si se tratara de exhibición de publicaciones, o la comprensión oral, si fuera escuchar una ponencia o clase en lengua extranjera, e incluso la expresión oral si el alumno quisiera dirigirse al ponente.

\subsubsection{El inglés para el desempeño laboral/profesional}

Esta sección considera el uso del inglés para el trabajo; teniendo en cuenta, de un lado, lo que se piensa sobre la perspectiva de la institución empleadora y, de otro, el punto de vista del alumno sobre el desempeño profesional. Las preguntas que exploranestos. púntos sonla 20 Q la $25 s$

Ítem 20: Es importante aprender inglés ya que los centros de trabajo lo re"Jorge Puccinelli Converso" quieren.

Ítem 25: El inglés no es muy necesario para mi carrera profesional.

Un $97.7 \%$ (68.2\% y $29.5 \%$ ) de los alumnos de San Marcos está de acuerdo con que el inglés es requisito de trabajo en las instituciones. (Ver Gráfico 4) Los otros alumnos del centro encuestados (31 sujetos) comparten la misma idea. De ellos, 30 muestran grados de acuerdo y únicamente 1 sujeto se muestra indeciso. Los profesionales tanto como los que no lo son coinciden en que los centros de trabajo esperan que sus empleados manejen el inglés.

Respecto del ítem 25, nótese que éste está planteado en términos negativos, destacándose en él que el idioma inglés no es necesario para la carrera 
profesional. La respuesta de los alumnos de pregrado fue de desacuerdo a dicho ítem. Cabe señalar que la manifestación de desacuerdo fue total, por el contrario ellos piensan, que esta lengua es necesaria para la carrera profesional.

Los otros alumnos del centro encuestados piensan igual en un $90 \%$, excepto los 5 trabajadores. Es posible que esta situación se plantee porque la pregunta no es adecuada para el grupo, ya que está dirigida a los de alumnos de carrera profesional.

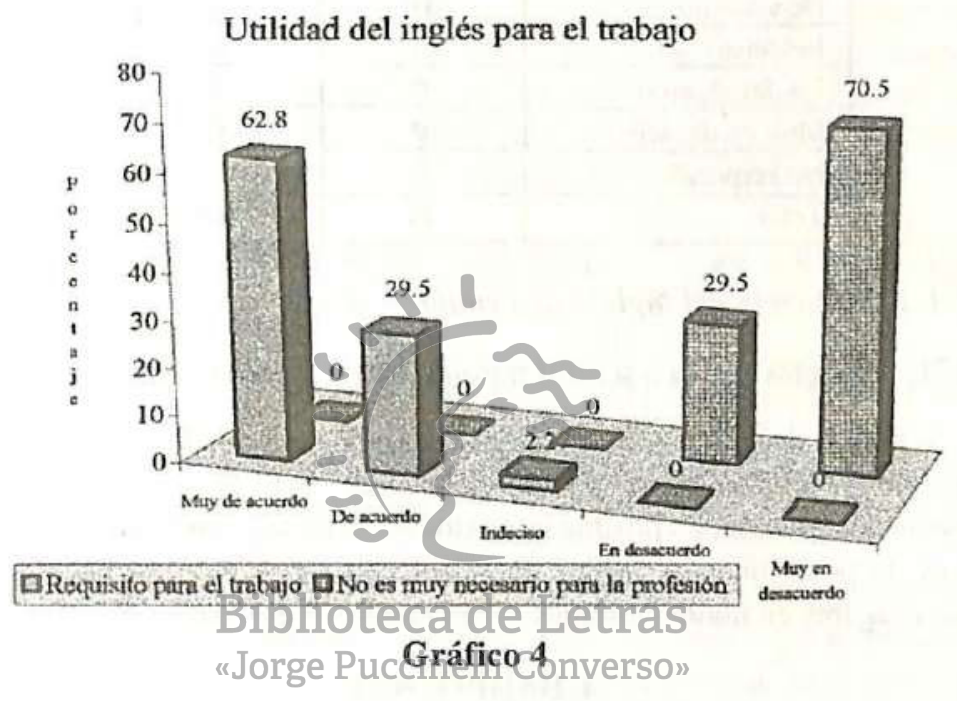

\subsubsection{Importancia del inglés para la actualización}

Esta sección considera la importancia del inglés para estar al día con los sucesos en el resto del mundo. Este punto está muy ligado tanto al estudio como al desempeño profesional. La concordancia con el enunciado es total en el caso de los estudiantes de San Marcos. (Ver Cuadro 1). En los otros grupos, de 31 sujetos estudiados, 29 están de acuerdo.

Item 23: El inglés permite estar al día con los acontecimientos mundiales, especialmente a través de Internet. 


\section{CUADRO 1}

Importancia del inglés para la actualización

estudiantes de pregrado - UNMSM

Ciclo 1 - Centro de Idiomas - 1998

\begin{tabular}{|c|c|c|}
\hline \multirow[t]{2}{*}{ Grado de aceptación } & \multicolumn{2}{|c|}{$\begin{array}{c}\text { Item } 23 \text { El inglés permite } \\
\text { Estar al día }\end{array}$} \\
\hline & Sujetos. & $\%$ \\
\hline Muy de a cuerdo & 34 & 77.3 \\
\hline De acuerdo & 10 & 22.7 \\
\hline Indeciso & 0 & 0 \\
\hline En desacuerdo & 0 & 0 \\
\hline Muy en desacuerdo & 0 & 0 \\
\hline No responde & 0 & 0 \\
\hline Total & 44 & 100 \\
\hline
\end{tabular}

\subsubsection{Importancia del inglés para viajar}

Item 21: El inglés me va a ser útil porque deseo viajar en el futuro.

Si bien la mayoría de alumnos de pregrado de San Marcos le ve utilidad al inglés para satisfacer su deseo de viajar, hay un $11 \%$ (5 personas) que no apoyan la declaración. Es posible que éstos no se hayan planteado, por distintas razones, la posibilidad de viajar fuera del país, o, si piensan hacerlo, no lo harían a lugares en dongẻ el inglés es-necesarioe (Ver Guadro $\mathrm{N}^{\circ} 2$ ).

\section{"Jorge CuADroli Nonverso"}

Utilidad del inglés para viajar

Estudiantes de Pregrado - UNMSM

Ciclo 1 - Centro de Idiomas - 1998

\begin{tabular}{|l|c|c|}
\hline \multirow{2}{*}{ Grado de aceptación } & \multicolumn{2}{|c|}{$\begin{array}{c}\text { Item 21 Utilidad } \\
\text { para viajar }\end{array}$} \\
\cline { 2 - 3 } & Sujetos & $\%$ \\
\hline Muy de acuerdo & 25 & 56.8 \\
\hline De acuerdo & 14 & 31.8 \\
\hline Indeciso & 4 & 9.09 \\
\hline En desacuerdo & 1 & 2.27 \\
\hline Muy en desacuerdo & 0 & 0 \\
\hline No responde & 0 & 0 \\
\hline Total & 44 & 99.96 \\
\hline
\end{tabular}


Los otros grupos a los que se aplicó la encuesta reaccionan de manera similar, excepto en el caso de los trabajadores en el cual un $80 \%$ (4 personas de un grupo de 5) no está seguro o está en desacuerdo con la declaración del ítem.

\subsubsection{Orientación integracionista}

\subsubsection{El inglés para la integración y la cultura}

Las preguntas de esta sección buscan medir la orientación integracionista de los alumnos. Obsérvese que los ítemes presentan grados de acercamiento a la otra cultura, desde la interacción con extranjeros para aprender de y sobre ellos (ítem 26), pasando por la posibilidad de hacer amigos (ítem 22), hasta una declaración extrema, ir a radicar a un país de habla inglesa (ítem 24).

Îtem 22 El saber inglés me abrirá la posibilidad de hacer amistad con personas de habla inglesa.

Ítem 24 Estudio inglés porque pienso irme a vivir a un país de habla inglesa.

Ítem 26 El saber inglés me va a perrnitir interactuar con extranjeros para conocer su cultura e idiosincrasia.

La idea que más aceptación tiene por los alumnos de San Marcos es la del ítem 26. Un $97 \%(40.9 \%)$ más $56.8 \%)$ opina qua Saber inglés le permitirá interactuar con extranjefosẹara coniocer șu eultura eqdiosincrasia. También un alto porcentaje, $86.4 \%$ (34.1\% más $52.3 \%$ ) está de acuerdo con el ítem 22 en que el inglés abre la posibilidad de hacer amigos. El ítem 24 no tiene mucha aceptación pues $56.79 \%$ están en desacuerdo con el enunciado de que estudian inglés porque piensan irse a radicar a un país de habla inglesa. De todos modos hay un $20,42 \%$ que manifiestan estar de acuerdo con este ítem. Es posible que, en parte, el estudiante esté influido por la situación económica del país, así como por el atractivo de ciertos países por su desarrollo científico (Ver Gráfico $\mathrm{N}^{\circ}{ }^{5}$ ).

Respecto a la opinión de los profesionales comprendidos en los otros alumnos del centro sobre ir a vivir a un país de habla inglesa, éstos tienen una posición algo diferente a los demás pues 5 personas, un $50 \%$ está de acuerdo, y 2 no se pronuncian en desacuerdo aunque sí indican no estar seguros de su posición. En cuanto a las preguntas 22 y 26 todos, los otros alumnos del centro tienen una opinión semejante a la del grupo de San Marcos. 


\section{Gráfico $\mathrm{N}^{\circ} 5$}

\section{El inglés para la integración y la cultura}

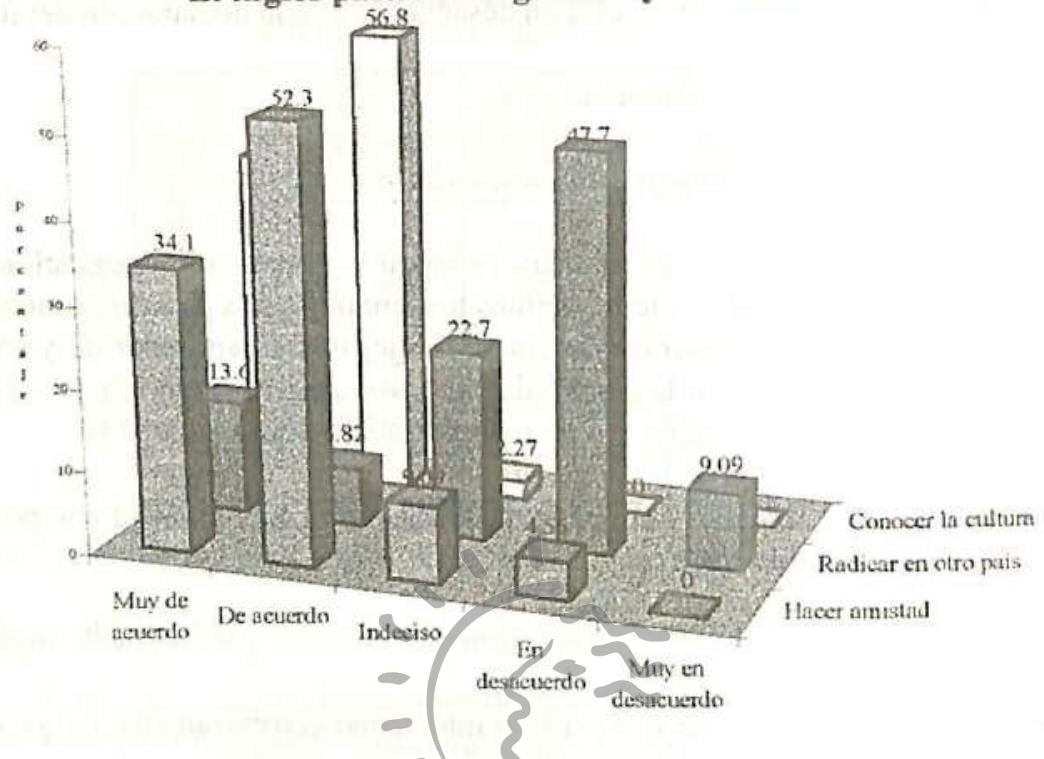

\subsection{Expectativas en relación al aprendizaje del inglés}

Las expectativas déūn alumngque voluñtariamente inicia sus estudios de idiomas pueden ser de distinto tipo. En este caso nos interesa el grado de dominio de la lengua que el "estudiante espera alcanzar, y lo que preferiría en cuanto a contenidos y metodología en los cursos.

\subsubsection{Nivel de dominio de la lengua inglesa}

Para tratar este aspecto se han planteado dos preguntas que incluyen dos distintos niveles de dominio de lengua, uno intermedio (ítem 28), y otro, avanzado. (ítem 31)

Ítem 28: Espero alcanzar un nivel intermedio de conocimiento del idioma inglés al finalizar mis estudios en este Centro.

Ítem 31: Al final de los cursos, espero dominar el inglés para hablarlo, leerlo y escribirlo. 
En cuanto a alcanzar un nivel intermedio de conocimiento del inglés, un $36.42 \%$ (16 alumnos) manifiestan indecisión o desacuerdo. Aparentemente un $63.6 \%$ (25\% y $38.6 \%$ ) está de acuerdo, pero observemos que esto no se confirma con las respuestas al otro ítem, el 31. (Ver Gráfico $N^{\circ} 6$ )

\section{Gráfico $\mathrm{N}^{\circ} 6$}

Expectativa de grado de dominio del inglés

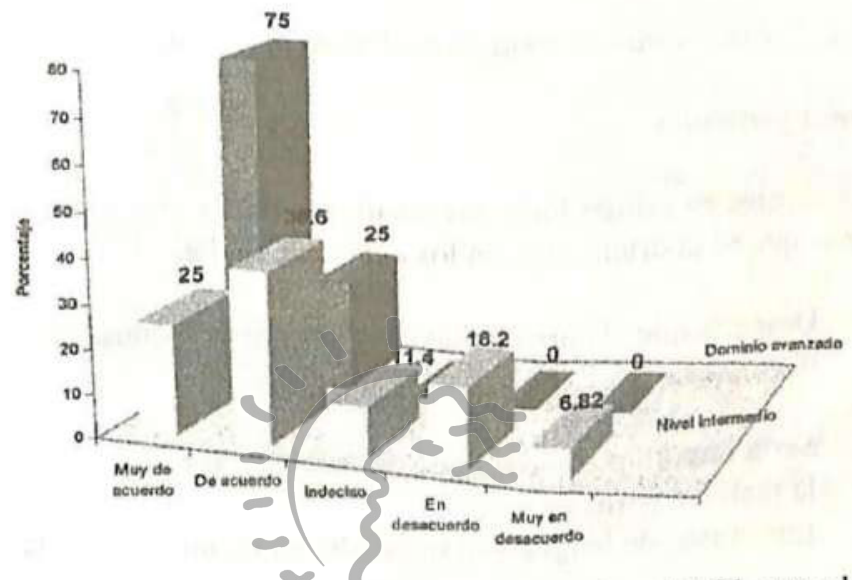

Contrastando las respuestas det f́tem 28 con las del 31 que plantea un nivel mayor de dominiob, vemos-que en estē último caso el $100 \%$ está de acuerdo con llegar a dominar el inglés. Más aún un $75 \%$ está "muy de acuerdo", es decir, es muy enfático. En este caso no hay indecision ni desacuerdo.

Parecería que en el ítem 31 algunos alumnos rectifican lo que contestan en el ítem 28. Sumando indecisión y desacuerdo en el ítem 28 , hay un $36.42 \%$. Estos alumnos que no manifiestan acuerdo seguramente son alumnos que tienen claro el nivel que desean alcanzar, no un nivel intermedio sino uno avanzado. Algo semejante sucede con el grupo de alumnos de las otras universidades, aquellos que no son de pregrado de San Marcos. En los grupos restantes que no son de San Marcos sí hay una diferencia, pues tanto para el nivel intermedio como para el avanzado el porcentaje de respuestas es igual. Se nota que al responder no se han detenido a pensar en la diferencia entre los niveles.

Podemos decir que nadie se opone a un alcanzar un dominio avanzado de la lengua, pero los estudiantes universitarios tanto de San Marcos como de las otras universidades tienen más conciencia del nivel que desean lograr. 
Los aspectos que se pueden incluir en un curso de lengua pueden ser de diverso tipo. ¿Cuáles son las preferencias de los alumnos respecto de este punto? Las preguntas del cuestionario se refieren tanto a temas generales (ítemes 29,30 y 32) y técnicos (ítem 33), como al desarrollo de habilidades lingüísticas (ítemes 33,34 y 38 ).

\subsubsection{Propuestas de temas para los cursos de inglés}

\section{- Temas generales}

Revisemos, en primer lugar los resultados de las preguntas que se refieren a temas que se podrían tratar en los cursos de inglés.

Ítem 29: Desearía que el curso incluyera temas de la cultura de países de habla inglesa.

Ítem 30: Sería importante que los cursos de inglés aborden algunos temas de la realidad peruana.

Ítem 32: Los cursos de lengua extranjera deben incluir temas de actualidad.

Los temas de actualjdad (ítem 32) son preferidos por un $93.1 \%(54.5$ y $38.6 \%$ ) del alumnado para ser-considerados en los cursos. Nadie se opone a la inclusión de dichos temaso (Ver Craficico Nio 7) Lodquie sí hay es algo de indecisión o duda, $6.82 \%$

Un porcentaje algo menor, un $89.9 \%(20.5 \%$ y $61.4 \%)$ se pronuncia a favor de incluir "algunos temas de la realidad peruana" (ítem 30). El desacuerdo es bajo, $4.55 \%$, aunque no así la indecisión, $13.6 \%$.

Un tema que alcanza menor acuerdo (ítem 29) que los anteriores es el relativo a la cultura de los países de habla inglesa. Están de acuerdo $72.7 \%$ (31.8\% y $40.9 \%$ ). La indecisión es alta, una cuarta parte (25\%) de los alumnos. Observamos que la indecisión va marcando diferencias entre los tipos de temas.

Los otros encuestados, los que no son del pregrado de San Marcos, están totalmente de acuerdo con incluir temas de actualidad. En cuanto a temas de la realidad peruana, la respuesta es semejante a la que dan los alumnos de San 
Marcos. La diferencia está en relación con el ítem 29, los otros grupos están de acuerdo con él en una proporción mayor que la de los alumnos de San Marcos, la cual va desde un $88 \%$ hasta un $100 \%$.

\section{Gráfico $\mathbb{N}^{\circ} 7$}

\section{Temas de los cursos de inglés}

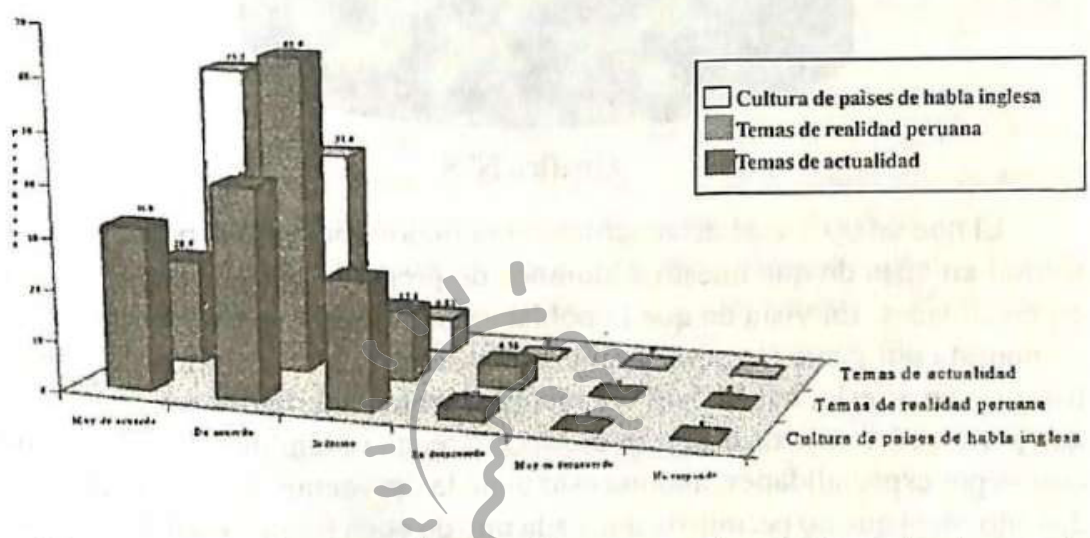

Vemos que en donde no hay problema es en lo relativo a los temas de actualidad. Pero, inclusive endơque se refiere ala cultura de los países de habla inglesa, si bien la dudaes altapel desacuerdo es bajo, $2.27 \%$. Lo que habría que estudiar más es la indecision que surge frente a estos tipos de temas.

\section{- Temas técnicos}

La pregunta 33 se refiere a vocabulario técnico, pero entendemos que éste no se puede trabajar fuera de contexto, por ello, este tipo de vocabulario nos lleva a tratar temas de especialidades.

Ítem 33: Los cursos deben incluir vocabulario técnico (comercial, turístico, científico, etc.)

Como vemos en el Gráfico $\mathrm{N}^{\circ} 8$ un $61.4 \%$ de los alumnos de pregrado de San Marcos está "muy de acuerdo" con el aprender vocabulario técnico y un $29.5 \%$, "de acuerdo", cantidades que sumadas dan un $90.9 \%$ a favor. (Ver Gráfico $\mathrm{N}^{\circ} 8$ ) 
Del otro grupo, los alumnos que no son de pregrado de San Marcos, al que se aplicó la encuesta sólo un sujeto se opone.

Temas tecnicos on los cursos do Ingios

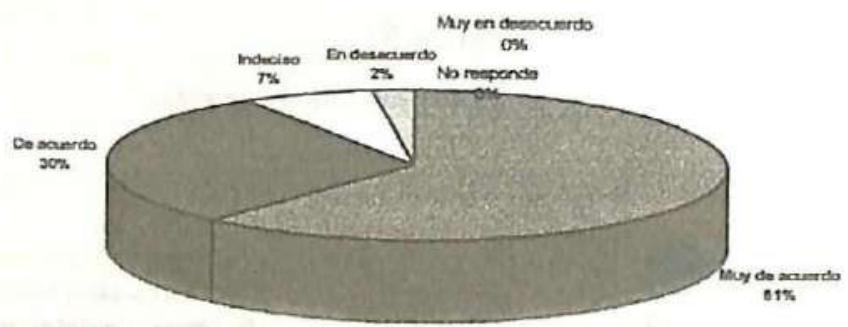

Gráfico $\mathrm{N}^{\circ} 8$

El que un $90 \%$ esté de acuerdo con la importancia del aspecto técnico es natural en vista de que nuestros alumnos de pregrado pertenecen a diferentes especialidades. En vista de que la población del Centro, del primer ciclo, está compuesta por gente interesada en distintas áreas y que no todos son especialistas o van a serlo, habría que ver planteamientos alternativos para ofrecer lo que parte de los sujetos desea en el aspecto técnico, también. Se podría dictar cursos por especialidades, aunque esto tiene la desventaja de unir a alumnos de distinto nivel que no permitiría que cada uno de ellos fuera atendido en el grado de desarrollo que le corresponde. Otra posibilidad es incluir algunas áreas técnicas dentro de los cursos, endistintas etapaseTambiểnsé podría hacer, en parte, un trabajo personalizadōorge Puccinelli Converso»

\subsubsection{Desarrollo de habilidades orales y escritas}

Tradicionalmente se ha pensado que en la universidad los alumnos necesitan el inglés para la lectura. En un sondeo que se hizo a los alumnos del Centro varios mencionaron que les interesaba el desarrollo de la conversación en inglés. Estos dos aspectos los observamos en las preguntas 34 y 38 que se han incluido en el cuestionario.

Ítem 34: Los cursos de inglés deben orientarse principalmente a la comprensión de lectura.

Ítem 38: Los cursos de inglés deben enfatizar el área de conversación en dicha lengua. 
La orientación del curso hacia el desarrollo de comprensión de lectura es pedida por 23 alumnos de pregrado de San Marcos o sea un 52.3\% (25\% más $27.3 \%)$. Veintiún alumnos $(47.67 \%)$ no apoyan esta orientación. (Ver Gráfico $N^{\circ}$ 9) Aquí se incluye $22.7 \%$ (10 alumnos) indecisos, $22.7 \%$ en desacuerdo, y $2.27 \%$ ( 1 alumno) muy en desacuerdo.

En cuanto a enfatizar la conversación, esto es, la lengua oral, 40 alumnos $(90.9 \%)$ se manifiestan a favor. De ellos 22, algo más del $50 \%$ están no sólo "De acuerdo" sino "Muy de acuerdo". Por otra parte, un alumno indeciso (2.27\%) y 3 en desacuerdo $(6.82 \%)$ se pronuncian en sentido contrario.

Las respuestas de los otros encuestados que no son del pregrado de San Marcos se dan en la misma línea. Sí les interesa la lectura, pero el porcentaje de respuestas a favor de enfatizar la conversación es mayor, un $100 \%$ en los profesionales y en los otros universitarios. En el caso de los trabajadores y egresados que aún no estudian ni trabajan el acuerdo es de un promedio de $78 \%$ para la conversación.

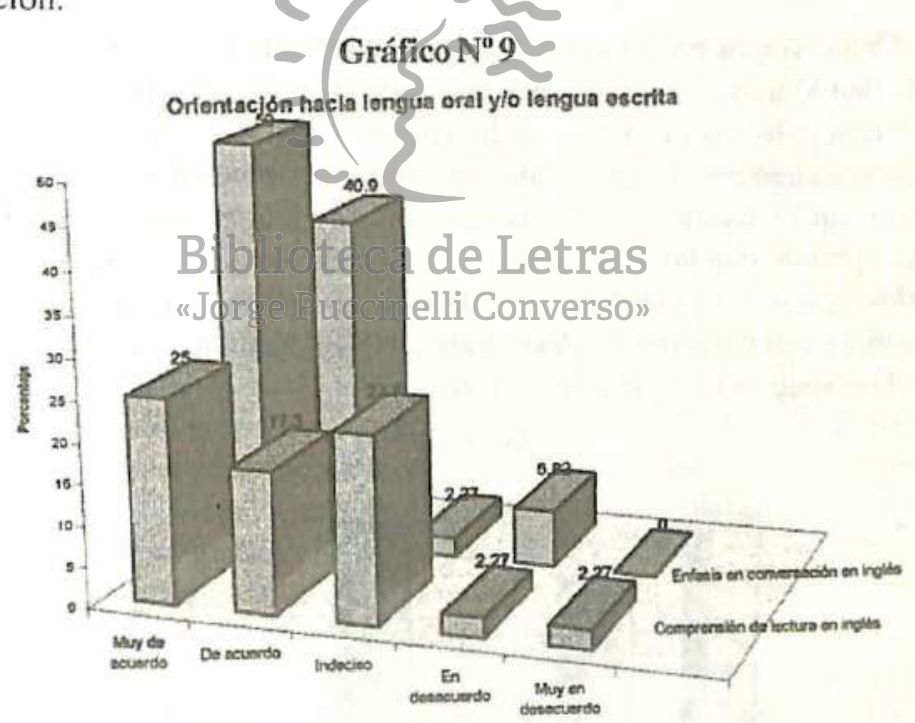

Está claro que los alumnos que asisten al Centro de Idiomas dan alta importancia a la lengua oral. La orientación hacia la lectura no implica dejar de lado la conversación en inglés. Los resultados respecto de la conversación en inglés se pueden relacionar con el desarrollo de comprensión oral, la cual puede ser necesaria para los eventos académicos. (Ver 3.1.1.1) 


\subsubsection{Opinión sobre metodología}

Los puntos que se incluyen en esta sección son algunos que significan un cambio con respecto al tratamiento tradicional de enseñanza de una lengua extranjera.

\subsubsection{Trabajo de grupo}

Ideas pedagógicas que se enfatizan actualmente y que están siendo llevadas a la práctica lentamente. son las que tienen que ver con el trabajo en grupo. Lo que sí es necesario es entender que hay distintas maneras de concebir lo que es trabajo grupal. Las preguntas que recogen información sobre este punto son la 35 y la 41 .

Ítem 35 No se aprende mucho de los otros estudiantes cuando se trabaja en grupo.

Ítem 41 Las clases deben incluir actividades realizadas en grupo.

Como vemos en el Gráfico $N^{\circ} 10$, un $84 \%$ (48\% más $36 \%$ ) de los alumnos de San Marcos consideran que las clases deben incluir trabajo en grupo. El $11.4 \%$ está indeciso y el $4.5 \%$, en desacuerdo (ítem 41 ). En relación con el otro ítem, o sea cuánto es lo que se aprende con este tipo de práctica, hay algo que destacar, un $75 \%$ están en desacuerdo o muy en desacuerdo con la idea de que NO se aprende mucho de ì iosi Otros estudiantesE En otfas palabras, consideran que sí se aprende. Sin embargo un 25\% hocomparteresta opinión y manifiestan su duda o su desacuerdo. (Ver Gráfico $\mathrm{N}^{\circ} 10$ ) Entre los otros grupos encuestados la respuesta es en el mismo sentido.

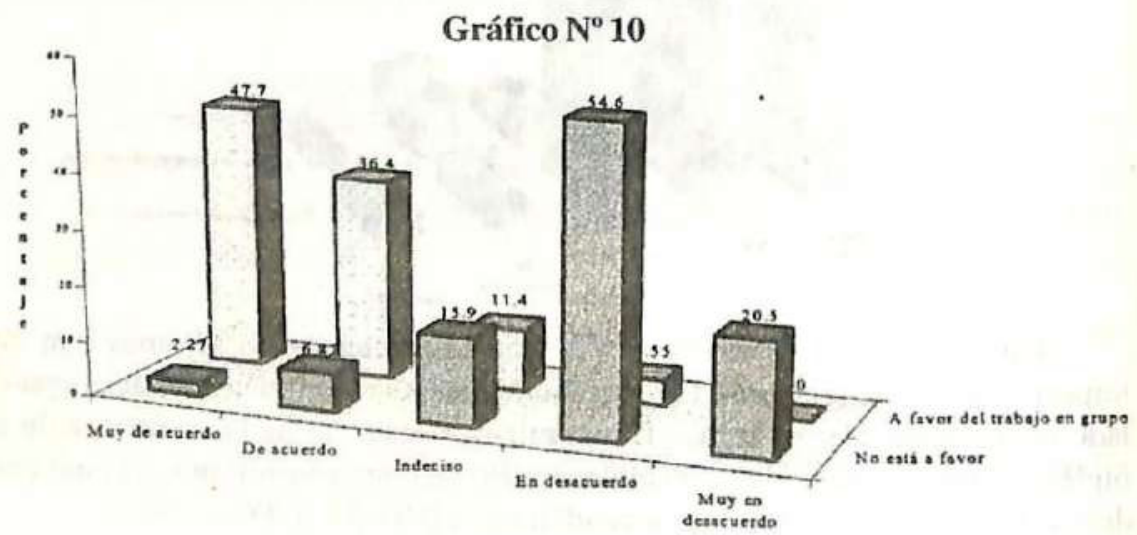


Parece que los estudiantes le dan un valor al trabajo cooperativo, en grupo, como parte de su aprendizaje. La duda de algunos podría tener que ver con la manera como se realiza el trabajo en grupo. Aquí se plantea la necesidad de una orientación pedagógica particular en el Centro y de la respectiva preparación del maestro.

\subsubsection{Lectura en lengua auténtica (no adaptada)}

Hay propuestas pedagógicas que destacan la necesidad de plantear retos al alumno que demanden un cierto esfuerzo por parte de él. Esto puede darse, por ejemplo, en los casos en que se le presenta material original, esto es, tal como se escribe normalmente. Generalmente se dan lecturas adaptadas y el estudiante, al enfrentarse a una publicación auténtica, se preocupa y no hace el intento de leerla. La inclusión de diversos tipos de impresos también permite variar los temas y adecuarlos a los diferentes estudiantes Los ítemes 40 y 43 tratan este punto.

Ítem 40 Los cursos de inglés deberían incluir otras publicaciones aparte del texto que se usa en el ciclo.

Ítem 42 Es una pérdida de tiempo leer revistas en inglés porque hay mucho vocabulario nuevo.

\section{Biblioteca de Ietras}

Los alumnos en un $80 \%$ (31 $8 \%$ mix de peuerdo y $47.7 \%$ de acuerdo) expresan acuerdo sobre la inclusión de otras publicaciones, pero suman un $20 \%$ (indecisos $15.9 \%$, en desacuerdo $4.55 \%$ ) quienes dudan o están en contra. Ninguno de los sujetos del grupo que no es del pregrado de San Marcos se opone a la idea de incluir otras publicaciones en los cursos de inglés. Los alumnos de San Marcos son los únicos que tienen un porcentaje de indecisión (15.9\%, o sea, 7 alumnos) y desacuerdo ( $4.55 \%$ ) que suma $20 \%$. Sin embargo, el porcentaje favorable a la lectura de revistas, esto es, de material auténtico, es $84 \%$ Obsérvese que el ítem 42 está planteado en términos negativos. Por ello, hay desacuerdo, $47.7 \%$, y fuerte desacuerdo, $36.4 \%$, cuando opinan sobre si es una pérdida de tiempo el leer revistas por la dificultad del vocabulario. También para este ítem la indecisión es alta, $11.4 \%$ (5 alumnos). (Ver Gráfico $\mathrm{N}^{\circ} 11$ ) De los otros grupos que no son de pregrado de San Marcos, sólo los egresados de secundaria están de acuerdo con la idea de que la lectura de revistas en inglés es una pérdida de tiempo por el vocabulario. 


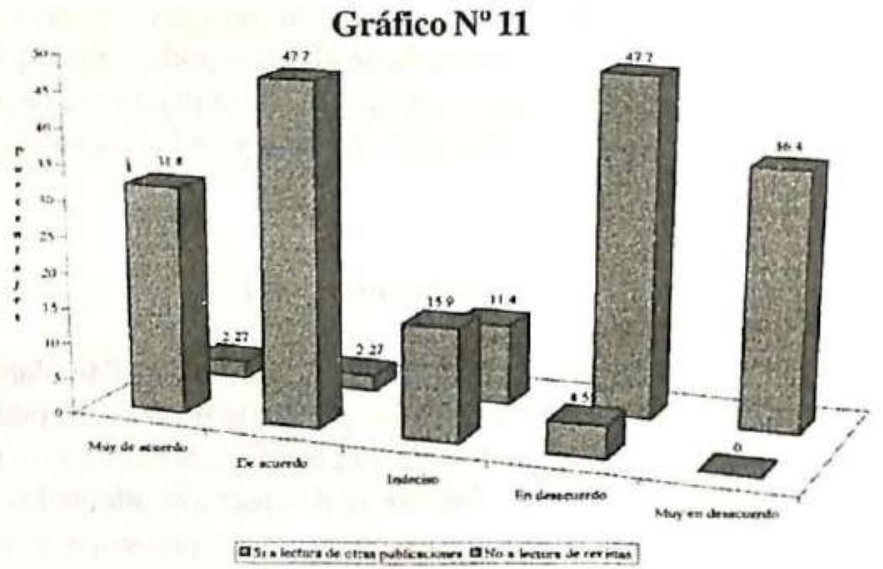

¿Tendrá que ver el resultado sobre la lectura de publicaciones auténticas con la mayor o menor autonomía para aprender que han desarrollado los estudiantes?

\subsubsection{Uso del inglés en situaciones reales}

Hay un interés de los estudiantes por una práctica del inglés tal como éste se usa realmente. Los ítemes 36,43 y 45 que tratan este aspecto, fueron tomados de lo planteado pos lós alumnos delCentrode Idiomas en el sondeo que se realizó al inicio del trabajo. Puccinelli Converso»

Ítem 36 Me gustaría que los cursos de inglés incluyan en su programa la comunicación con extranjeros (Internet, correo convencional).

Ítem 43 Se debería incluir como parte del curso de inglés charlas de personas de habla inglesa.

Ítem 45 Los cursos de inglés, además de ejercicios, deben incluir también otro tipo de trabajos e investigaciones.

El grupo de San Marcos desea que se le proporcione la oportunidad de practicar el inglés en situaciones reales. Por ello, observamos que en el ítem 36 un $90.9 \%$ declara estar de acuerdo con incluir en el programa de inglés formas de comunicación con extranjeros aunque un $9.09 \%$ se declara indeciso. De otro lado, las respuestas al ítem 43 sobre recibir charlas de hablantes de inglés 
tienen un $86.3 \%$ de acuerdo, en los dos grados de aceptación. Otra idea es practicar el inglés, en parte, con algunos trabajos más relacionados con las labores propias de un universitario como plantea el ítem 45: "otro tipo de trabajos e investigaciones". Un porcentaje alto está de acuerdo, pero éste es un poco más bajo en comparación con los otros ítemes, $67.76 \%$ (20.06\%, muy de acuerdo, y 47.75, de acuerdo). El porcentaje de indecisión es bastante alto, 27.3\%. (Ver Gráfico $N^{\circ} 12$ )

En el caso de los otros estudiantes del centro, los que no son del pregrado de San Marcos, sucede algo semejante, se da una alta proporción de acuerdo con los ítemes 36 y 43, y menor acuerdo con el ítem 45.

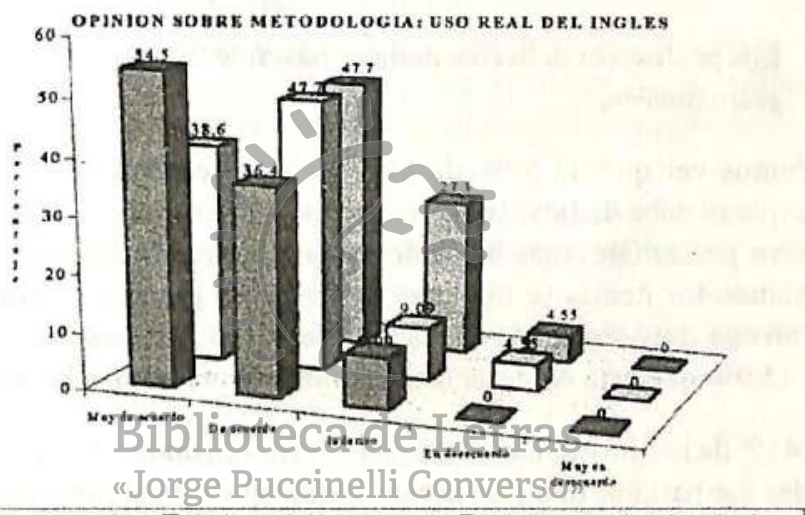

Q Comunieación con extranjeres DCharlas con bablaslex de ingles Q El ingles en trabajes e investigeciunes

\subsubsection{Teoría gramatical}

El papel de la gramática en la enseñanza-aprendizaje de lenguas extranjeras es un tema de controversia entre los especialistas. Es interesante conocer qué piensa un alumno -no especialista en áreas vinculadas a esta lengua-que está comenzando sus estudios de inglés en el Centro de Idiomas y que trae una experiencia de aprendizaje de esta lengua de la secundaria. Obviamente, si está en un Ciclo 1, es que lo que aprendió antes no le permite usar el inglés como él quisiera. No estamos poniendo en duda que el aprendizaje de gramática sea necesario. La pregunta es hasta qué punto dedicarle tiempo y cuánto trabajo teórico realizar. Por ello se incluyó el ítem 44. (Ver Gráfico $\mathrm{N}^{\circ} 13$ ) 


\section{Gráfico $\mathrm{N}^{\circ} 13$}

Bastante tiempo para explicación de gramática

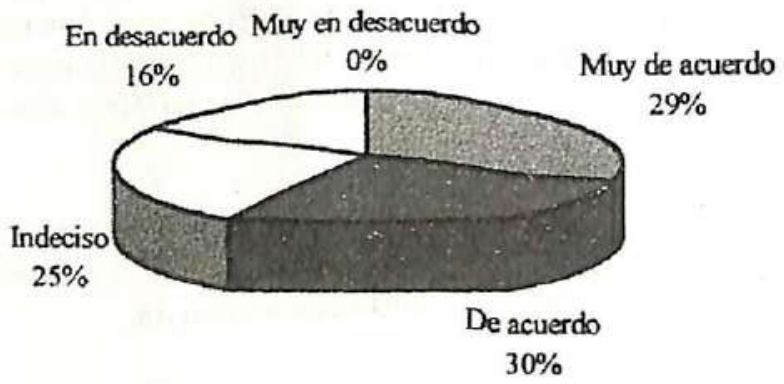

Ítem 44 Los profesores deberían dedicar bastante tiempo a explicar las reglas gramaticales.

Podemos ver que un $59 \%$ de los alumnos de pregrado de San Marcos considera que se debe dedicar bastante tiempo a la explicación gramatical. Este es uno de los porcentajes más bajos de acuerdo al que hemos tenido en todo el estudio cuando los ítemes se plantean en términos positivos. Además, hay un $25 \%$ que no está muy seguro de que la idea del enunciado sea lo que ellos desearían y un $15.9 \%$ que está en desacuerdo, ambos, porcentajes bástante altos.

Un $41 \%$ de los universitarios no estáa favor (en đưda, $25 \%$ y en desacuerdo, $16 \%$ ) de dedicar bastante tiempoala explicación gramatical por parte del profesor. El conocimiento sobre el sistema de la lengua es necesario para quien aprende inglés, pero hay que probar maneras alternativas de enseñarlo. Maneras más orientadas al uso, a la aplicación de este conocimiento, y no a largas explicaciones. Lo que podría explicar el $59 \%$ de acuerdo es que los alumnos hayan experimentado un desequilibrio entre lo teórico y lo práctico en su aprendizaje de idiomas anterior al del Centro de Idiomas y por eso la mayoría no se pronuncie a favor de dedicar regular tiempo a explicaciones gramaticales.

\section{Conclusiones}

\subsection{Población del Centro de Idiomas}

Para efectos de planificación del programa del Centro de Idiomas de la Facultad de Letras de la Universidad Nacional Mayor de San Marcos, un pri- 
mer aspecto que debemos tener en cuenta es que este centro atrae alumnado que no sólo está constituido por estudiantes de la misma universidad. Del estudio realizado en el $77 \%$ de los alumnos asistentes del primer ciclo del nivel básico del Centro, se establece que el $57 \%$ eran estudiantes de pregrado de San Marcos y $42.85 \%$ en su mayoría eran de fuera.

Los 33 alumnos que no son estudiantes de San Marcos, y en su mayoría provienen de fuera de la universidad, realizan una variedad de actividades. Un $54.54 \%$ son del área profesional $(30.30 \%$ son profesionales y $24.24 \%$ estudiantes de otras universidades), siguen los egresados de secundaria, $24.24 \%$; los trabajadores $15.15 \%$ y $6.06 \%$ de alumnos de secundaria.

Los 44 estudiantes de pregrado de San Marcos estudiados pertenecen a distintas áreas. Las que predominan son la de Matemática y Física con $32 \%$ del conjunto, y luego la de Ciencias Económicas, $16 \%$. Los demás sujetos se distribuyen en pequeños grupos entre Ciencias Biológicas, Médicas, de la Educación, Humanas, Sociales y Políticas.

En cuanto a la edad, estos alumnos sonjóvenes. El 93\% es menor de 29 años.

Como podemos ver es una variada población la que hay que atender en el Centro de Idiomas.

\subsection{Orientación

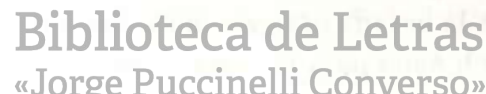

Las razones para aprender inglés con las que se manifiestan de acuerdo los estudiantes de pregrado de San Marcos son principalmente de carácter instrumental, o sea, con fines utilitarios. Al pronunciarse a favor de ellas los alumnos tienen una posición más enfática que para las de carácter integracionista, seleccionando en mayor número la opción "Muy de acuerdo" en vez de "De acuerdo"

Las razones, en orden de porcentaje de aceptación son: la necesidad del inglés para la profesión (100\%), para acceso a la información bibliográfica $(100 \%)$, para cumplir con un requisito del centro de trabajo $(97.7 \%)$, para eventos académicos $(95.5 \%)$, y para viajar $(88,6 \%)$.

En general, las respuestas de los alumnos del centro que no son de San Marcos son semejantes a las del pregrado de nuestra universidad, excepto en el caso de los trabajadores cuando se refieren a la utilidad del inglés porque de- 
sean viajar. El $80 \%$ de estos están en desacuerdo con el ítem. Es posible que no consideren esta posibilidad debido a los compromisos de trabajo adquiridos y a la situación económica.

Cuando los estudiantes de San Marcos responden a las preguntas de orientación integracionista, si bien el acuerdo es alto en dos de ellas las respuestas no son tan enfáticas. La alternativa "Muy de acuerdo" es menos elegida. El orden según el porcentaje de aceptación es de $97 \%$ de acuerdo con el ítem sobre la posibilidad que da el inglés para interactuar con extranjeros y conocer su cultura e idiosincrasia, y de $86.42 \%$ de acuerdo con la idea de que el inglés abre la posibilidad de hacer amigos entre la gente de habla inglesa. Hay un ítem que tiene poca aceptación: la idea de que estudian inglés para irse a vivir a un país de habla inglesa. Sólo un $20.42 \%$ acepta esta idea. Sin embargo comparando a los alumnos del pregrado de San Marcos con el grupo de estudiantes que ya son profesionales, el $50 \%$ de estos se manifiesta de acuerdo con que estudia para irse a radicar a alguno de estos países

La utilidad del inglés para acceder a bibliografía siempre ha sido reconocida, particularmente en el ambiente uniyersitario. No así la utilidad del inglés en eventos académicos, idea con la que también están de acuerdo los alumnos. ¿Qué eventos académicos en inglés se dan en nuestro medio? Una posibilidad es ponencias o clases en inglés en la modalidad de teleconferencias. Esto nos llevaría a la necesidad de läjēngua oral, en su modalidad de discurso académico, distinto del uso de la lengua para la comunicación cotidiana.

Como era de esperar los estudiantes se pronuncian más a favor del aprendizaje del inglés para fines instrumentales, esto es, utilitarios o prácticos. En cuanto a los tres ítemes de carácter integracionista planteados que presentan desde un acercamiento a la cultura y gentes de países extranjeros, pasando por hacer amistad con personas de habla inglesa, hasta llegar a la idea de radicarse en un país de habla inglesa, hay acuerdo con respecto a los dos primeros. Podemos decir, entonces, que los alumnos sí le ven valor a la lengua inglesa para comunicarse con otros grupos culturales, favoreciendo así la relación intercultural.

\subsection{Expectativas}

En lo referente al grado de dominio del inglés que se espera alcanzar, un $100 \%$ de los alumnos de San Marcos espera dominar el inglés para hablarlo, 
leerlo y escribirlo. Sólo un $32 \%$ de ellos tienen clara la idea de niveles de manejo de lengua. Los estudiantes de otras universidades también responden igual, no así los demás sujetos que parecen no haber reflexionado respecto del grado de dominio que desean lograr.

\subsubsection{Contenidos y habilidades}

\section{Temas}

Los temas que prefieren los alumnos de San Marcos para los cursos de idiomas son, en primer lugar, los de actualidad (93.1\%), luego algunos temas de la realidad peruana $(89.9 \%$ ), y temas de cultura de países de habla inglesa (71.3\%). Además, más de un $90 \%$ está a favor de incluir vocabulario técnico el cual estaría relacionado con temas de distintas especialidades

\section{Habilidades}

Otros contenidos preferidos tiener que ver con el desarrollo de habilidades lingüísticas. Sólo un $52.3 \%$ de los alumnos de pregrado de San Marcos está de acuerdo con orientar los cursos a la lectura en inglés. En cambio, un $90 \%$ está a favor de enfatizar la conversación. De los demás grupos a los que se aplicó la encuesta, el acuerdo con el poner énfasis en la conversación es en los profesionales y estudiantès de otras uniyersidades mayor aún, es de $100 \%$.

\subsubsection{Metodología}

\section{"Jorge Puccinelli Converso"}

\section{Trabajo en grupo}

Una de las técnicas que propone la metodología de enseñanza actualmente es el trabajo en grupo. Un $75 \%$ piensa que sí se aprende de otros miembros del grupo cuando se trabaja de este modo, pero un $25 \%$ lo duda. De modo semejante piensan los universitarios que no son de San Marcos. Probablemente esto esté relacionado con la didáctica que emplea el profesor.

\section{Material auténtico}

Otro principio educativo nos orienta a no darle todo listo al alumno sino plantearle retos para que se esfuerce dentro de sus posibilidades. Un ítem donde se consulta esta idea es el que se refiere a practicar lectura no sólo con el 
libro de texto, el cual está graduado, sino con otras publicaciones. Un $80 \%$ de los alumnos de San Marcos están de acuerdo con este planteamiento, mientras los demás encuestados lo están en un $100 \%$. También se consulta sobre la lectura de revistas en inglés que traen vocabulario nuevo. Los alumnos de San Marcos en un $84 \%$ y los demás sujetos estudiados, excepto los egresados de secundaria, consideran que vale la pena hacer el esfuerzo para leer estas revistas, manifestando que esto no es una pérdida de tiempo.

\section{Contacto con hablantes nativos de inglés}

Los alumnos están de acuerdo con cursos en donde se programen algunas actividades en donde se establezca contacto a distancia (correo convencional o electrónico) o directo (charlas de invitados) que permitan comunicarse con interlocutores hablantes de inglés. En estos casos, los estudiantes de San Marcos indican acuerdo de $90 \%$ para las actividades a distancia y de $86.3 \%$ para las directas. Esto nos refiere a un punto ya mencionado, el de la utilidad del inglés para eventos académicos, los cuales pueden ser ponencias o charlas que hay que escuchar.

\section{Aplicación al trabajo universitario}

No les es tan interesante en este momento, Ciclo 1 del Nivel Básico, pensar en aplicar el inglés èn otros tipos de trabajos personales, por ejemplo, en investigación. Esta otra posibilidad de aplicación del ingles es apoyada por un porcentaje significativo aunque no tan alto como en otros casos, $67.78 \%$. La duda es alta, un $27.3 \%$. En los otros grupos la respuesta es semejante.

\section{Explicaciones gramaticales}

El conocimiento sobre el sistema de la lengua, sobre su estructura es necesario para quien aprende inglés, pero hay que probar maneras alternativas de enseñarla. Un 59\% de los alumnos de pregrado de San Marcos aceptan que se dedique bastante tiempo a explicar la gramática, pero un $40.9 \%$ no apoya este punto de vista. En otras palabras, un alto número de estos alumnos no está de acuerdo con esta manera de enseñar gramática. Se podría considerar alternativas más orientadas al uso, a la aplicación de este conocimiento y no a largas explicaciones, a dedicarle mucho tiempo a la teoría. Los otros grupos en cambio, excepto los universitarios, están de acuerdo en dedicar bastante tiempo a la explicación gramatical, tratamiento que es bastante tradicional. 
Se observa entonces una tendencia hacia aspectos de enfoques metodológicos modernos por la mayoría de alumnos estudiados, y aunque hay un menor porcentaje de alumnos un tanto tradicionales, la institución necesita considerar alternativas para ver el tratamiento más adecuado que permita tener en cuenta los estilos de aprendizaje de los adultos.

El Centro de Idiomas de la Facultad de Letras y Ciencias Humanas tiene un conjunto de alumnos muy variado, con orientación predominantemente instrumental, muy motivado, y que preferiría un tratamiento que incorpore ideas recientes sobre contenidos y métodos en el aprendizaje de idiomas.

No se puede tomar decisiones de planificación con sólo el punto de vista del alumno, pero éste sí nos puede servir para relacionarlo con los puntos de vista de los docentes y de las autoridades. Un buen profesor de idiomas, mejor dicho un maestro, siempre podrá encontrar maneras de tomar en cuenta al alumno, en otras palabras, de personalizar su enseñanza.

BABBIE, EarlR.

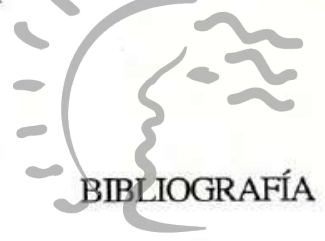

1988

Métodos de investigación por encuesta. Fondo de Cultura Económica.

BAKER, Colin

1988

Key Issues in Bilingualism and Bilingual Education. Multilingual Matters Ltd. Philadelphia.

LOZADA, Minnie, ALONZO Alicia y REYES, Johanna

1997 Percepción de la utilidad de conocer idiomas según alumnos de pregrado de la Universidad Nacional Mayor de San Marcos. (Documento de investigación sin publicar, CILA, UNMSM)

NUNAN, David

1989 El diseño de tareas para la clase comunicativa. Cambridge University Press. 
NUNAN, David

1992

Research Methods in Language Learning. Cambridge University Press.

POZZI-ESCOT, Inés y LOZADA TRIMBATH, Minnie Eloísa

1989 ¿Qué razones para aprender inglés tienen los alumnos de secundaria de colegios estatales?". En López, Luis Enrique, y otros, Temas de Lingüistica Aplicada. CONCYTEC.GTZ, Lima.

RICHARDS, Jack C. y LOCKHART, Charles

1994

Reflective Teaching in Second Language Classrooms. Cambridge University Press.

WILLIAMS, Marion y BURDEN, Robert L.

1997

Psychology for language teachers. United Kingdom: Cambridge University Press.

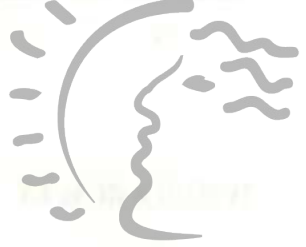

Biblioteca de Letras

"Jorge Puccinelli Converso" 